\title{
Modelling Biological Evolution: Developing Novel Approaches
}

\author{
Andrew Morozov ${ }^{1}$
}

Published online: 15 October 2019

(c) Society for Mathematical Biology 2019

Mathematical modelling is known to be a powerful and convenient tool to explore different aspects of biological evolution (including evolution of human society) and explain the existing genetic complexity of the real world. It is now well recognised that a satisfactory understanding of natural selection and inheritance, origin of complex patterns of animal behaviour, evolution of infectious diseases, and brain functioning is simply impossible by only laboratory experiments and field observations. Theoretical models allow a wide-range exploration of a biological problem and sometimes can even provide an alternative to biological experiments which can hardly be done at all. Currently, mathematical and computational approaches are being rapidly developed to allow us to cope with the existing and newly emerging challenges in biological evolution. However, hundreds of theoretical works are now being published each year on the topic, and it is often hard to avoid getting lost in such an immense flux of publications. The aim of the present issue of the Bulletin of Mathematical Biology is to provide a useful guide to important recent developments in some key areas in modelling biological evolution. In particular, the following topics are presented: (1) theoretical approaches to modelling selection in systems with inheritance; (2) role of stochasticity (demographic and environmental) in biological evolution and survival of populations; (3) understanding the efficiency of brain functioning and neural learning; (4) impact of spatial and time scale on evolution; (5) social dilemmas. We should emphasise that the papers in the issue are not limited to only one of the above topics, but rather lie at the intersection of them.

Most of the works presented here are in fact contributed papers from the international conference 'Modelling Biological Evolution: Developing Novel Approaches' (MBE 2017), which took place in Leicester, UK, on April 2017 and brought together about 150 theoreticians and empirical evolutionary biologists with the main aim of creating debates and productive discussions.

Among the other conclusions, this special issue emphasises the need for a more constructive dialogue between theoreticians and experimentalists.

Andrew Morozov

am379@leicester.ac.uk

1 Department of Mathematics, University of Leicester, University Road, Leicester LE1 7RH, UK 
The first two papers of the issue by Chalub and Souza (2019) and Broom et al. (2019) present recent advances in evolutionary game theory involving a large number of players. Chalub and Souza (2019) explore links between $d$-player game theory and fixation probability, i.e. the probability that the frequency of a particular trait in the population will eventually become unity. Note that understanding the fixation probability is a central aspect of evolution modelling. Unlike the previous works, the authors address the inverse problem: from a particular fixation pattern and the population size, they try to define the game being played by the considered population. Chalub and Souza (2019) explore fixation in a Wright-Fisher model and demonstrate that evolutionary fitness can be approximated by a $d$-player game in the case the number of players is large enough. Importantly, the corresponding pay-off matrix can provide information about interaction patterns among individuals within the population. The authors use both analytical and extensive numerical methods to support their insightful conclusions.

The study of Broom et al. (2019) revisits the important class of evolutionary games known as social dilemmas characterised by cooperation and defection. (Cooperation benefits the entire group, whereas defection benefits only one individual.) Progress in social dilemmas will be necessary for better understanding of the evolution of altruistic or cooperative behaviour in animals and humans (Nowak 2006). Important practical applications include cooperative hunting and sharing resources by animals and humans. Unlike the previous works on social dilemmas, M. Broom and co-authors consider the simulation with non-fixed group size in the game: a variable size would be a more realistic feature of animal populations in the wild as opposed to a fixed-size game. The considered approach will also allow for a richer variety of social dilemmas. For social dilemmas with variable group size, Broom et al. (2019) found a rather counterintuitive effect: an increase in the variability in group sizes for non-threshold public goods games would generally be favourable for the evolution of cooperation, which is not necessarily true for threshold public goods games.

The next contributions to the issue, by Kuzenkov and Morozov (2019) and Sandhu et al. (2019), aim to revisit mathematical approaches to modelling natural selection in generic self-replicating systems with strong inheritance. The theoretical study by Kuzenkov and Morozov (2019) suggests a novel framework to model evolution in an arbitrary function space of inherited strategies (applicable to model both scalarvalued and function-valued traits). A new concept of evolutionary fitness is proposed based on the idea of the ranking order of competing strategies: such ranking compares long-term dynamics of measures of sets of inherited units in the space of strategies. The authors formulate the variational principle of modelling selection which states that selection in the space of strategies should eventually maximise evolutionary fitness. Kuzenkov and Morozov (2019) then obtain expressions for evolutionary fitness for various models including those with age structuring and delay, which was previously a challenge. The related study by Sandhu et al. (2019) extends the theoretical ideas of Kuzenkov and Morozov (2019) and proposes a novel computational method to determine evolutionarily optimal life-history traits in selfreplicating systems with strong inheritance. The need for an efficient computational method comes from the fact that in many cases it is hard to obtain an analytical expression for evolutionary fitness for an arbitrary model (Morozov and Kuzenkov 
2016). The main idea of the method of Sandhu et al. (2019) is the reconstruction of evolutionary fitness using the underlying model equations of population dynamics. Interestingly, under some assumptions, the computational method can be used even in the case where we only have population dynamics time series, i.e. without the underlying model. As an insightful biological example, Sandhu et al. (2019) explore optimal patterns of diel vertical migration (DVM) of zooplankton in the water column, which is generally considered to be the largest synchronised movement of biomass on Earth (Hays 2003). Unlike some previous studies on the topic, the authors consider both scenarios of DVM with static and dynamic predators. The model predicts that in waters with high food abundance of food for zooplankton, the dynamic predation results in an increase in the amplitude of DVM, which is not observed in the case of static predators, where DVM generally abruptly ceases.

The following three studies by Dissanayake et al. (2019), Reisch and Langemann (2019), and Gidoin and Peischl (2019) emphasise the role of space in modelling evolutionary advantage and selection. In particular, Dissanayake et al. (2019) explore genotypic selection in a spatially heterogeneous environment under stoichiometric constraints. They show that spatial heterogeneity allows coexistence of competing genotypes via stable equilibria which are not possible in a well-mixed model. As the study case, Dissanayake et al. (2019) consider interactions in a plankton community under different levels of eutrophication which is a practically an important research area in applied ecology (Morozov et al. 2019 and references therein). In their work, Reisch and Langemann (2019) investigate the role of space in the shift of a liver infection from an acute to a chronic disease type which can be seen as an evolutionary advantage for infected individuals. Using the reaction-diffusion framework, the authors demonstrate that infection chronification is promoted by the immune response of the organism and evolution would favour a medium-level efficiency of $\mathrm{T}$ cells. Gidoin and Peischl (2019) highlight the striking similarity between species range expansion in ecology and the spatiotemporal dynamics of tumour growth within the host. The authors argue that the intra-tumour heterogeneity is of crucial importance during the development of solid tumours; in particular, this concerns accumulation of deleterious mutations at the edge of the expanding range as well as enhancement of dispersal abilities of abnormal cells. This paper provides an interesting and a not yet well-studied so far connection between the previous observations of accelerating of the dispersal rate in structured populations in ecology (Petrovskii et al. 2008) and clinical/theoretical studies of solid tumour development.

The work by Vitale and Kisdi (2019) contributes to the long-standing debate regarding evolutionary suicide previously raised in the literature (Matsuda and Abrams 1994; Parvinen and Dieckmann 2013), i.e. when adaptive evolution of a species can lead to its extinction. Vitale and Kisdi (2019) reconsider the seminal paper by Matsuda and Abrams (1994) by applying the critical function analysis technique as well as relaxing some main assumptions of the original model. The authors show that in a broader community model including three species (one predator and two prey systems) evolutionary suicide becomes possible via some new scenarios, for example via a fold bifurcation of limit cycles or an indirect mechanism of consecutive invasions of prey species. The study of Cropp and Norbury (2019) considers eco-evolutionary interac- 
tion in a system with mutualism: a typical ecological application includes pollination of plants by animals. Mathematically, the authors use a Lotka-Volterra modelling setting and apply a genetic computational algorithm to simulate mutual adaptation of the plant and pollinator populations. The main question that the authors address in the paper is about the probability to observe plant/pollinator mutualist niches.

The next two contributions to the issue highlight the importance of stochasticity and environmental change in biological evolution and population extinction. In their study, Orive et al. (2019) explore how populations should adapt to a gradual environmental shift of the phenotypic optimum to be able to persist which is known as evolutionary rescue (Gomulkiewicz and Holt 1995). The evolutionary rescue has recently received much attention in the research literature. M. Orive and co-authors apply an individual-based simulation model and a mean-field analytic model to explore the role of population size, type of reproduction (sexual/clonal), genetic variation, and the rate of environmental change on the possibility of evolutionary rescue. The modelling results show that larger populations generally exhibit better survival properties. The authors find a larger vulnerability to extinction in clonal rather than sexually reproducing populations. Interestingly, the extinction of populations in the model due to environmental changes generally occurs unexpectedly without a clear early warning signal. The work of $\mathrm{Yu}$ and $\mathrm{Li}$ (2019) considers extinction in finite populations due to weak demographic stochasticity. The authors are interested in the expected time of extinction. They compare the efficiency of two major frameworks borrowed from theoretical physics such as the Wentzel-Kramers-Brillouin (WKB) and the FokkerPlanck methods. (These methods were originally applied in statistical mechanics and quantum mechanics.) Yu and Li (2019) apply both methods to some well-known population dynamics models such as logistic growth, endemic SIR, predator-prey, and competitive Lotka-Volterra model. The authors show that in models having a stable equilibrium or a limit cycle, where extinction is caused by a large amplitude rare events, the WKB method would be more appropriate too. However, in the case of marginally stable states, where extinction is driven by Gaussian fluctuations, the Fokker-Planck approximation can be more straightforward.

Finally, an insightful study by Tyukin et al. (2019) explores a fundamental problem of modern neuroscience-codifying memories. The main focus of the authors is on understanding the efficiency of functioning of the human brain as compared to that of other mammals. Using a simple model, the authors show that neuronal cells can selectively detect and learn arbitrary information items in the case they operate in a high-dimensional space. The mathematical model can predict a remarkably high selectivity of neurons to a particular type of information and the possibility of fast dynamical learning of neurons. Interestingly, the model demonstrates that neurons are capable of associating and learning uncorrelated information items. Mathematically, the modelling results are based on the recently reported stochastic separation theorems and the concentration of measure in high-dimensional spaces (Gorban and Tyukin 2018). These results will contribute to the basic concepts of static and dynamic memories of the human brain functioning. This study will be also relevant to explain the existing complexity in the social life of humans. 
Acknowledgements We would like to thank the authors, reviewers, and the Chief Editor Professor Alan Hastings for their collaboration in producing this special issue of the Bulletin of Mathematical Biology.

\section{References}

Broom M, Pattni K, Rychtáŕ J (2019) Generalized social dilemmas: the evolution of cooperation in populations with variable group size. Bull Math Biol. https://doi.org/10.1007/s11538-018-00545-1

Chalub FA, Souza MO (2019) From fixation probabilities to d-player games: an inverse problem in evolutionary dynamics. Bull Math Biol. https://doi.org/10.1007/s11538-018-00566-w

Cropp R, Norbury J (2019) Simulating eco-evolutionary processes in an obligate pollination model with a genetic algorithm. Bull Math Biol. https://doi.org/10.1007/s11538-018-0508-1

Dissanayake C, Juan L, Long KR, Peace A, Rana MM (2019) Genotypic selection in spatially heterogeneous producer-grazer systems subject to stoichiometric constraints. Bull Math Biol. https://doi.org/10.1007/ s11538-018-00559-9

Gidoin C, Peischl S (2019) Range expansion theories could shed light on the spatial structure of intra-tumour heterogeneity. Bull Math Biol. https://doi.org/10.1007/s11538-018-00540-6

Gomulkiewicz R, Holt RD (1995) When does evolution by natural selection prevent extinction? Evolution 49:201-207

Gorban AN, Tyukin IY (2018) Blessing of dimensionality: mathematical foundations of the statistical physics of data. Philos Trans R Soc A Math Phys Eng Sci 376(2118):20170237

Hays G (2003) A review of the adaptive significance and ecosystem consequences of zooplankton diel vertical migrations. Hydrobiologia 503(1):163-170

Kuzenkov O, Morozov A (2019) Towards the construction of a mathematically rigorous framework for the modelling of evolutionary fitness. Bull Math Biol. https://doi.org/10.1007/s11538-019-00602-3

Matsuda H, Abrams PA (1994) Timid consumers: self-extinction due to adaptive change in foraging and anti-predator effort. Theor Popul Biol 45:76-91

Morozov AY, Kuzenkov O (2016) Towards developing a general framework for modelling vertical migration in zooplankton. J Theor Biol 405:17-28

Morozov A, Denaro G, Spagnolo B, Valenti D (2019) Revisiting the role of top-down and bottom-up controls in stabilisation of nutrient-rich plankton communities. Commun Nonlinear Sci Numer Simul 79:104885

Nowak M (2006) Evolutionary dynamics, exploring the equations of life. Harward University Press, Cambridge

Orive ME, Holt RD, Barfield M (2019) Evolutionary rescue in a linearly changing environment: limits on predictability. Bull Math Biol. https://doi.org/10.1007/s11538-018-0504-5

Parvinen K, Dieckmann U (2013) Self-extinction through optimizing selection. J Theor Biol 333:1-9

Petrovskii S, Morozov A, Li BL (2008) On a possible origin of the fat-tailed dispersal in population dynamics. Ecol Complex 5:146-150

Reisch C, Langemann D (2019) Modeling the chronification tendency of liver infections as evolutionary advantage. Bull Math Biol. https://doi.org/10.1007/s11538-019-00596-y

Sandhu KS, Morozov AY, Kuzenkov O (2019) Revealing evolutionarily optimal strategies in selfreproducing systems via a new computational approach. Bull Math Biol. https://doi.org/10.1007/ s11538-019-00663-4

Tyukin I, Gorban AN, Calvo C, Makarova J, Makarov VA (2019) High-dimensional brain: a tool for encoding and rapid learning of memories by single neurons. Bull Math Biol. https://doi.org/10.1007/s11538018-0415-5

Vitale C, Kisdi E (2019) Evolutionary suicide of prey: Matsuda and Abrams' model revisited. Bull Math Biol. https://doi.org/10.1007/s11538-018-0472-9

Yu X, Li XY (2019) Applications of WKB and Fokker-Planck methods in analyzing population extinction driven by weak demographic fluctuations. Bull Math Biol. https://doi.org/10.1007/s11538-018-04836

Publisher's Note Springer Nature remains neutral with regard to jurisdictional claims in published maps and institutional affiliations. 